\title{
Guest editorial
}

\section{Introduction to the special issue on disrupting the status quo}

It is our great pleasure to present this collection of papers authored by participants of the 8th International i-Rec Conference "Disrupting the status quo: Reconstruction, recovery and resisting disaster risk creation”, held at the University of Florida, Gainesville, 5-8 June 2019 [1]. In the context of massive global challenges caused by rapid human development, a group of scholars and practitioners from around the globe gathered to discuss some of the most pressing but under-represented, misunderstood and divisive topics in disaster studies. We collectively asserted that status quo practices, policies and solutions are not getting us where we need to go. As a consequence of this limited vision, we are missing opportunities for positive transformational change during disaster recovery and reconstruction.

Despite a rhetoric of benevolent intent, it has become clear that efforts towards disaster risk reduction are simply not stemming the tide of new risk being created. As we pursue business as usual (growth and profit), the crisis of inequality - and the resulting risk creation is deepening, both between countries and inside borders. While international frameworks and agreements are met with plaudits, many scholars argue that planetary boundaries are rapidly being approached or exceeded and that natural systems simply cannot support "development" at the pace observed since the 1950s.

This special issue makes the case that disaster risk creation has become the norm within our current political, economic and societal systems. Overconsumption and the accumulation of power and resources are promoted by design. Inequality is fuelled by greed, profiteering and a deliberate attempt to amass power. If disaster risks are to be reduced, this needs to change. Unfortunately, the most opportunistic at these times are adherents to the status quo. The papers that follow take up the challenge of how to position ourselves for positive change at the necessary time.

In adaptations of full papers submitted at i-Rec 2019, the authors present compelling visions of disrupting the status quo. Whilst exploring different challenges located in different contexts, the common thread of this special issue is the authors' attention to the Disaster Risk Creation paradigm. Authors broadly examine disasters with a root cause lens and embed their arguments in a nuanced understanding of a global productive system and capitalist societies. They bring frequent attention to the underrepresented, the marginalized and the oppressed, who are disproportionately affected by disasters. Most papers touch upon the role of neoliberalism in disaster risk creation. Sandoval et al. in particular focus on disaster capitalism and explain how political reforms can hinder or enhance this manifestation of neoliberalism.

The role of community is salient in this special issue. Hendriks and Opdyke show how community-based self-recovery in the absence of technical housing assistance can aid disrupting authority of experts. They demonstrate the power of community strength and solidarity while advocating for effective ways of spreading technical knowledge into local practice. Leite and Ribeiro discuss how large infrastructure development projects violate the rights of indigenous peoples by destroying local culture and the environment, and call for disrupting state profiteering. Ajulo et al. build this argument for local autonomy and ownership, highlighting the potential of localisation and degrowth as a way to reduce vulnerability.

The papers also focus on narratives that surround reconstruction and recovery. Cheek shows how the rhetoric of community-based or participatory approaches to reconstruction rarely match the reality of implementation. His research disrupts elite interests by unmasking the agendas that would use words as legitimising tools. Lizarralde et al. emphasise that the

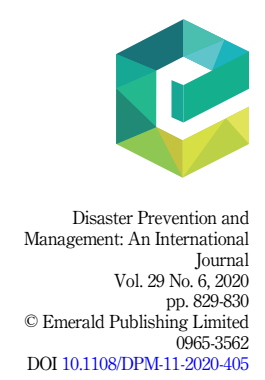


DPM

29,6 language of "experts" grounded in terms or concepts largely adopted by academics and NGOs does not support our understanding of local struggles for social justice, but instead reestablishes the status quo. Waldmueller extends this theme, arguing that mainstream disaster narratives cannot generate the required knowledge that would allow us to understand risk that is permanently neglected. Often risk is created because of our lack of engagement with local people actually experiencing oppression and violence, and our failure to recognise who is creating risk.

Discussing the technical aspects of reconstruction are similarly important as it helps disrupt the cycle of replicating experiences and problems. Vaccari et al. demonstrate the importance of transitional shelter for internally displaced people who face daily risks and live through everyday disasters on an ongoing basis. Feng and Toshiya discuss the idea of sponge cities as an approach to tackle urban flooding that has become prominent in China in recent years. Both papers, however, highlight that technological decisions on their own cannot reduce disaster risks.

What all these papers highlight is that a disruption of normative disaster narratives and positions offers an opportunity to reconsider how we do - and should do - disaster research. The perspectives emerging from this special issue open up difficult conversations for the disaster studies community, but also pathways for moral, ethical and politically and socially salient research.

We would like to thank all the paper authors and reviewers for making this special issue possible, as well as the i-Rec community of researchers and practitioners for responding to a "disruptive" conference theme with passion and vision. We would also like to thank the University of Florida's College of Design, Construction and Planning, M.E Rinker, Sr. School of Construction Management and Florida Institute for Built Environment Resilience (FIBER) for supporting and hosting the 2019 i-Rec conference.

Jason von Meding

Florida Institute for Built Environment Resilience, University of Florida, Gainesville, Florida, USA

Ksenia Chmutina Architecture, Building and Civil Engineering, Loughborough University, Loughborough, UK

Giuseppe Forino

Department of Environmental Sciences, University of East Anglia, Norwich, UK, and

Emmanuel Raju

Global Health Section, Copenhagen Centre for Disaster Research, University of Copenhagen, Frederiksberg, Denmark

\section{Note}

1. The conference themes have been discussed on the Disasters: Deconstructed Podcast, Season 1 Episodes 9 and 19. 